\title{
Base editing a CRISPR way
}

A way to gene edit without double-stranded DNA breaks is now entering labs.

\author{
Vivien Marx
}

B ase editors have pounced onto the geneediting stage. Labs working on plants, microbes, human cells, and model organisms are using and rapidly advancing these tools ${ }^{1-3}$. Base editors use facets of CRISPR-Cas9-based systems by combining enzymes that modify DNA with, for example, catalytically inactive Cas9 variants. With base editors, labs can convert one base to another: a C.G base pair becomes T.A, or A.T is converted to G.C.

This nucleotide conversion happens without double-stranded cutting by fully active CRISPR-Cas9 nuclease, without invoking DNA repair mechanisms that follow double-stranded breaks or using a donor template. Proponents say base editing is an easier way to deliver editing machinery to cells, and some evidence suggests base editing makes fewer unwanted insertions and deletions than classic CRISPR-Cas9.

It's been only two years since the first base-editing publication from the lab of Harvard University researcher David Liu, who says it's been "amazing and humbling" to see such quick adoption in the research community. Base editing has "completely changed the way we build models in the lab," says Weill Cornell Medicine researcher Lukas Dow. He hopes base editing will help labs build more accurate cancer models to expand on information from large-scale tumor sequencing. "We've taken pretty blunt tools to date to understand the genetics of cancer," says Dow. Many cancer mutations are highly recurrent singlenucleotide changes and some genes have hot-spot mutations waiting to be studied in detail. Gene deletion is an experimental option but, for example, mutations within the $p 53$ gene show activity unlike wholegene deletion. Such insight matters for understanding tumor behavior and drug response, he says.

Base editors are pushing into the clinical realm for somatic cell treatments. Work with human embryos is also under way. One research group altered a mutation that causes $\beta$-thalassemia using cloned human embryos made from a patient's cells $\mathrm{s}^{4}$. A separate team achieved, in their words, "genetic correction" in human embryos heterozygous for a mutation underlying

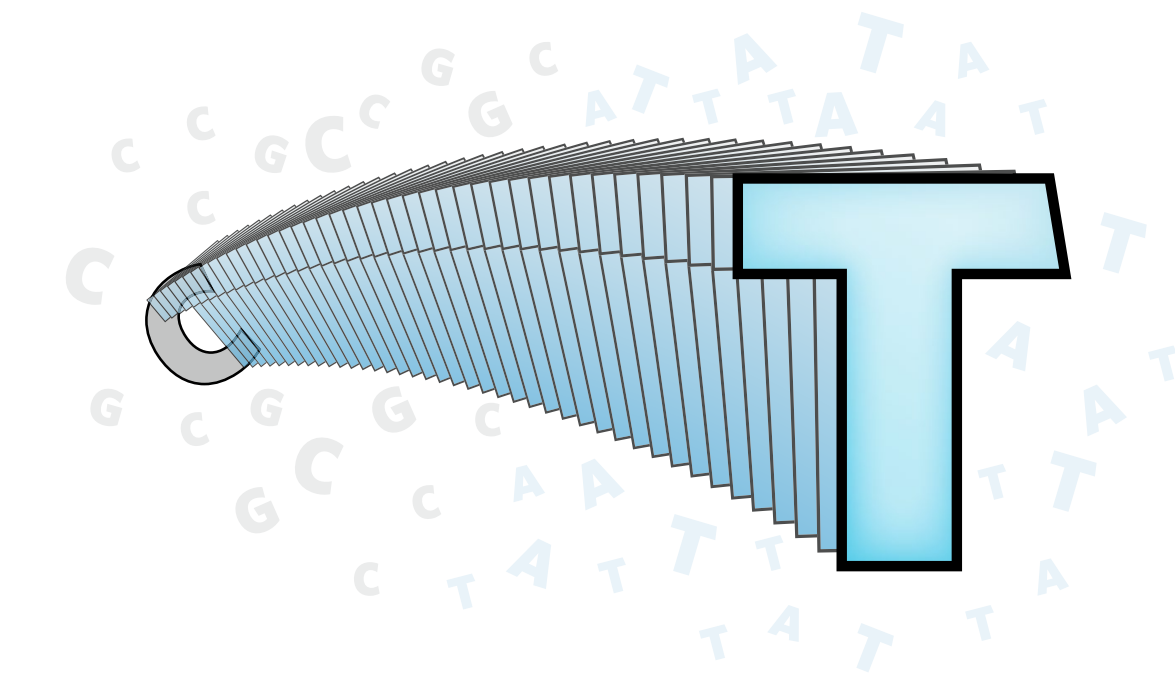

Base editors are new genome-editing tools that convert nucleotides without double-stranded breaks. The first base editors converted cytosine (C) to thymine ( $T$ ).

Marfan syndrome 5 . As with CRISPR-Cas9, ethics discussions are likely to accompany developments in base editing.

As gene-editing tools are refined and improved, possible therapeutic applications nudge closer and "the importance of quantifying rare offtarget mutagenesis has increased," note the developers of CRISPResso2, a sequence-analysis software tool updated to quantitatively analyze base-editing experiments, as well as allele-specific data. Within a site targeted for base editing, multiple cytosines or adenines can be modified, and the tool quantifies events at each of these nucleotides, says CRISPResso co-developer J. Keith Joung, a Massachusetts General Hospital (MGH) researcher.

Along with Liu and Feng Zhang from the Broad Institute of MIT and Harvard, Joung co-founded Beam Therapeutics. The hope is, says Liu, to quickly, thoughtfully, and safely explore how base editing might help patients with genetic diseases with few, if any, treatment options. The three scientists have also co-founded Pairwise Plants, which focuses on agricultural applications with gene editing, including base editing.

\section{Make a point mutation}

According to Addgene curator Brook Pyhtila, 23 CRISPR-Cas9 plasmids designed for base-editing plasmids were deposited in 2016, 37 in 2017, and 87 in 2018 as this issue of Nature Methods goes to press. This year, 2,100 requests for base-editing plasmids have come in, for a total of 3,600 since 2016.

In the CRISPR field, Addgene has helped labs iterate, optimize and reoptimize tools, says Dow. The use of traditional material transfer agreements likely would have slowed things down. His lab has deposited many base-editing plasmids

Depositions of base-editing plasmids

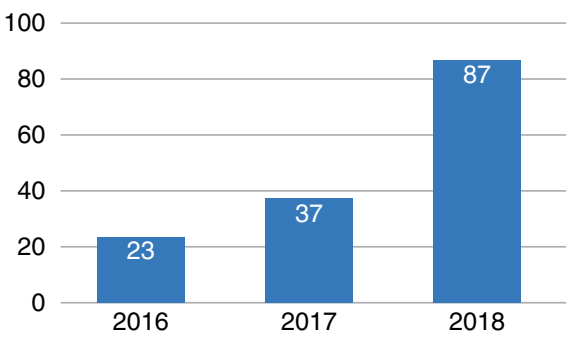

The number of CRISPR Cas plasmids designed for base editing is rising as the field grows. (Source: Addgene) 


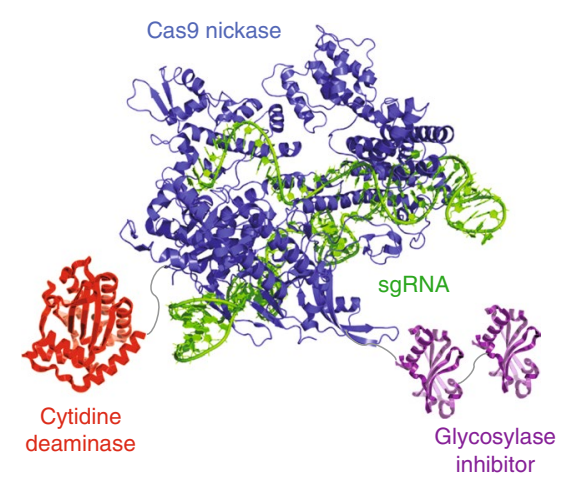

Base editors, here the general structure, use some aspects of CRISPR systems for nucleotide conversion. (Liu lab, Harvard Univ.; adapted with permission from ref. ${ }^{1}$ )

with Addgene but he is concerned that this wide choice might confuse labs new to base editing. Perhaps, he says, developers can add suggestions or information about applications. More online resources will likely help newcomers to the field of base editing, he says. Given his lab's CRISPRCas9 experience, he says the group can readily see when one guide RNA (gRNA) for base-edit targeting might work better than another. BE-Designer, a tool not from his lab, is the first of many sgRNA design tools for base editing likely to appear, he says. At the same time, even beautiful gRNAs fail for no clear reason, he says. Stanford University researcher Michael Bassik also shares baseediting-related plasmids on Addgene. It leads to interactions with users and kicks off collaborations. He shares plasmids pre-publication to give labs quick access to updated tools.

CRISPR-Cas9-based systems can be used for single-nucleotide editing, but that involves a double-stranded break. Base editing, which uses Cas9 variants alongside cytidine deaminases, leverages the ability

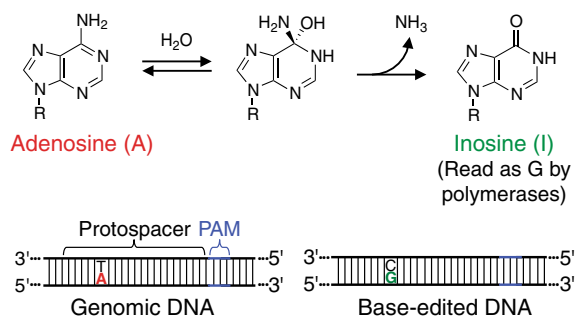

The Liu lab also engineered adenine base editors, which can convert A.T to G.C. No natural enzyme could do this, so the team evolved the needed deaminase. (Adapted with permission from ref. ${ }^{3}$ ) of Cas9 to unwind double-stranded DNA to target base conversion. Editors come in a number of flavors. As Alycia Bittner, a member of Addgene's technical support team, explains, the first generation of base editors from the Liu lab uses cytidine deaminase APOBEC1 fused to catalytically impaired Cas9 (dCas9). Each next generation of base editors from the Liu lab improved editing efficiency or specificity or both. Second-generation editors from his lab, BE2, added a base-edit repair inhibitor, a glycosylase inhibitor, to the fusion protein. The third generation of editors, BE3, uses a Cas9 nickase (Cas9n) instead of dCas9. Cas9n makes single-strand cuts, or 'nicks', in DNA, and the edit is propagated by harnessing of a cell's repair mechanism. Fourth-generation base editors, BE4, involve modifications such as an additional copy of a repair inhibitor.

The Liu lab also engineered adenine base editors (ABEs), which in Liu's view are the most useful ones in that they can convert $\mathrm{A} \cdot \mathrm{T}$ to $\mathrm{G} \cdot \mathrm{C}$, and thus can revert the most common mutation in living systems. To develop ABEs, the team evolved the needed deaminase, as nature provides no enzyme that can convert an A into something resembling a $\mathrm{G}$, says Liu.

\section{Seeking efficiency}

There is no efficiency level below which an editor is useless or above which an editor is useful for all applications, says Liu. The metric depends on the target, the intended application, and the downstream biology. A low base-editing efficiency might suffice for labs generating a point mutation in a crop, but to achieve therapeutic benefit in a person a high degree of correction in a target organ might be needed, accompanied by a high base-editing efficiency.

When labs try base editors, says Dow, they need to assess how a version with higher fidelity might have lower efficiency. For a lab isolating clones, efficiency might be less of a concern. In a pooled setting, scientists can increase cell numbers to find the base-edited cells they need.

For base-editing experiments in prokaryotes that use a glycosylase inhibitor, efficiency is near $100 \%$ and robust, says Kobe University researcher Keiji Nishida. But, when overexpressed, the inhibitor can cause nonspecific mutations, which is why he and his colleagues are working on a base-editing method without this inhibitor. As more experimental data are collected, efficiency patterns might emerge, he says. Certain base editors might perform similarly

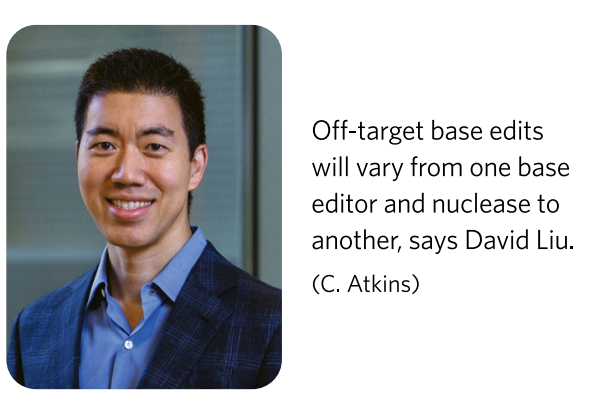

among higher eukaryotes such as plants and animals, lower eukaryotes such as yeast and microbes, or prokaryotes.

Helpful assays for assessing base-editing experiments include fluorescence reporters, says Liu, and an "occasionally overlooked hero" is high-throughput sequencing. Sequencing works well for measuring baseediting-induced mutation frequency, says Bassik. But when labs create a mutant and select for it, "that's not really an accurate way of quantifying mutation frequency," he says. His team also uses fluorescent reporters for rough impressions about a new base editor, but this is also not a raw mutation frequency readout. "There's definitely plenty that can be done to improve the ability to detect these things," he says.

The ideal window for base-editing activity is related to the number of protospacer-adjacent motifs (PAMs) in the genome that can be targeted, says Liu. A window width of one base is ideal, but that's the "extreme case" in which it's experimentally possible to target every possible site in the genome. In practice, says Liu, most of the current set of base editors offer activity windows with widths between two and five nucleotides. Taken together with the set of PAMs that can be targeted with base editors, this offers a "good chance" of targeting a base pair of interest.

Some of the alternative or expanded PAM Cas9 variants from his and other labs can work with base editors, "but often, under non-ideal conditions, their activities are lower than that of wild-type Cas9-derived base editors," says Liu. That leaves room for new Cas-protein variants and corresponding base editors that maximize the base-edit targeting scope, he says.

\section{Bystanders, off-targets}

Bystander and off-target mutations are hard to quantify, says Bassik, but need careful consideration. The occurring off-target edits will vary from one base editor and nuclease 


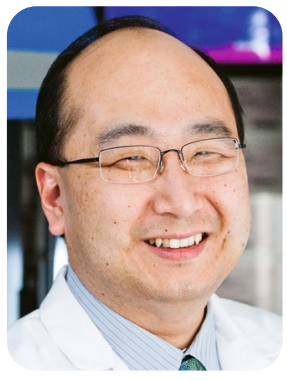

By mining the diversity

of cytosine deaminases and using protein engineering, scientists can make base editors with desired traits, says Keith Joung.

(MGH Photography)

to another, says Liu. Base editing does not lead to the types of insertions, deletions, translocations, and rearrangements that follow CRISPR-Cas9 double-stranded breaks, but base editing can lead to off-target point mutations. "For reasons we don't entirely understand, the locations of offtarget base editing appear to be a subset of the locations of off-target nuclease-mediated genome modification," says Liu. His lab and others are looking into this.

When a bystander such as a nearby nontargeted $\mathrm{C}$ or $\mathrm{A}$ is altered, it might not be worrisome, says Liu-for example, when an edit is intended to disrupt a gene with an early stop codon to prevent production of a pathogenic protein or destroy a regulatory sequence. If a bystander base is in a protein-coding gene, labs should work out whether the change is deleterious. A base editor with a five-base editing window will typically change only one amino acid. A change might also be "silent" and not change an amino acid. As Joung explains, the Liu lab narrowed the editing window with an engineered cytosine deaminase, APOBEC1. Sometimes, says Joung, this decreases base-editing activity in that narrowed window. Base editors with narrowed windows or context-specific ones, such as those from the Joung lab, improve odds, says Liu. "I think developing a large set of context-specific base editors is one of the most promising new frontiers in the field."

When the Joung lab set out to increase base-edit precision in a non-narrowed window that contains multiple cytosines, they looked at cytosine deaminases in human and other mammalian cells ${ }^{6}$. One of them, APOBEC3A (A3A), deaminates cytosines and has a preference for modifying cytosine that is preceded by a thymine. To Jason Gehrke, a member of the Joung lab who joined Beam Therapeutics this summer, this preference seemed a good base-editor trait. Gehrke tethered A3A to Cas9n to target a cytosine preceded by thymine and assessed the base edit with GFP. But in the tethered configuration, the base editor's preference went missing: it worked equally well when $\mathrm{C}$ was preceded by $\mathrm{T}$ and when it was not.

Perhaps, the team mused, the result was due to a higher local concentration of the deaminase. They weakened the affinity of A3A for DNA, for example, by altering the parts that interact with the DNA backbone. Gehrke studied the structure of A3A to determine which residues interact with which part of the DNA, mutated these residues, tested them, and found which ones restored the cytosine positional preference, leading to a more precise way to base edit in an editing window with multiple Cs, says Joung. Their enhanced A3A mutants (eA3A) also showed less off-target base editing.

Overall, says Joung, the work shows how labs can mine the natural diversity of cytosine deaminases and, coupled with protein engineering, develop base editors with desired traits. Tools will keep emerging as labs enter the base-editing field. A genomic site now hard or even impossible to edit might become editable, he says.

\section{Microbes, plants}

Nishida, along with colleagues from Tokyo University of Science, developed deaminasemediated targeted nucleotide editing (TargetAID) for base editing in Escherichia coli ${ }^{7}$. The editor is a cytidine deaminase, PmCDA1, fused to nuclease-deficient CRISPR-Cas9, and its point mutations did not hinder cell growth. Even though CRISPR-Cas9 is derived from the bacterial immune system, conventional gene editing in prokaryotes is often quite toxic, says Nishida. Base editing appears to be less toxic but he and his colleagues had to reduce the amount of base-editing protein. Among other facets, they used weak promoters and introduced a protein-degradation tag and a glycosylase inhibitor. The inhibitor stops removal of uracil created by the deamination, and the protein-degradation tag reduces overall protein concentration in the cell.

Their method can be used in different ways, such as with a nickase instead of dCas9, and with or without a glyocosylase inhibitor. Nishida recommends labs test base editors to find the one right for their organism and application. The nickase boosts base-editing efficiency in eukaryotes but it's somewhat toxic to prokaryotes, he says. He recommends assuring that gRNA expression is high. The gRNA length also matters for experimental success. "We believe that the base editing is applicable to wider range of organisms than nucleasebased genome editing," he says.

For point-mutagenesis experiments, plant biologists have expanded their base-editing toolbox beyond the conversion of cytosine to thymine. Scientists at several Korean institutions adapted ABEs from the Liu lab to mediate the conversion of A.T to G.C in protoplasts of Arabidopsis thaliana and Brassica napus with high efficiency ${ }^{8}$. Separately, researchers in China modified ABEs to make A.T-to-G.C edits in wheat plants and rice ${ }^{9}$. Among their tweaks was to optimize the position of the tRNA adenosine deaminase to the nCas9.

\section{High-throughput}

Liu says his group is collaborating with several labs on high-throughput baseediting projects. "Since base editors can be readily reprogrammed with guide RNAs just as Cas9 can, the same principles apply and early results are quite promising," he says. Nishida and his colleagues, too, are working on high-throughput base-editing approaches. "Hopefully in the future we can do genome-wide mutagenesis at single-base resolution," he says.

At Stanford University, Bassik uses base editing to deploy "mutagenesis machinery" for the "diversifying base editors" he built together with postdoctoral fellow Gaelen Hess and colleagues. Such editors are also in development, for example, in the lab of Xing Chang at Shanghai Institutes for Biological Sciences and Shanghai Jiao Tong University School of Medicine. The Bassik lab tiles mutagenesis machinery across a full protein-coding region using variants of cytosine deaminases engineered to be hyperactive and multiple gRNAs. They did this with PSMB5, the drug target of the chemotherapeutic bortezomib, says Bassik. The team targeted the gene and found known and previously unknown mutations that confer resistance to the drug. In this way,

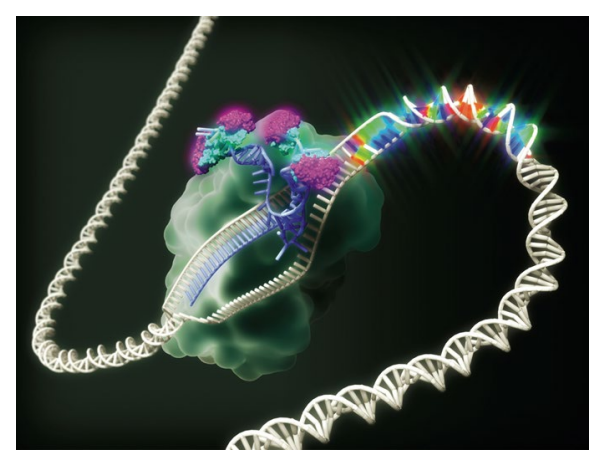

With diversifying base-editing, many mutations are created at a targeted site, which helps to explore, for example, drug resistance. (J. Heras, Equinox Graphics; Bassik lab, Stanford Univ.) 
base editing helps with characterizing mechanisms of chemo resistance.

The team consulted protein crystal structure data of PSMB5 in complex with bortezomib and saw that some of the mutations were in the drug-binding site. Even when a lab lacks structural data, the diversifying base-editing method can be useful for mapping resistance mutations, says Bassik. The method is not slated to be introduced into patients but it can be used to find potential new therapeutic targets, he says.

The team has applied this approach to human cells and it has been used in zebrafish and other model systems, he says, including plants for crop engineering. Unlike random mutagenesis with bacteria using chemicals or radiation that leads to mutations all across the genome, the method provides a way to generate mutations at a select genomic location and increases the chances of recovering potential mutations at that site.

Bassik sees his method as easier than error-prone PCR followed by a library of plasmids that are introduced into an experimental system at nonendogenous levels. Potential applications include labs looking for a library of mutants to evolve new protein or enzyme function, or to study drug resistance, an area of active research in academic and biopharmaceutical industry labs. Speaking more generally, Bassik says that applications with base-editing tools are "limited only by your imagination."

\section{Optimizing}

Plenty of cells are difficult to transfect, says Dow. He and his team addressed issues related to the enzyme expression for geneediting experiments ${ }^{10}$. Expression is one reason that much work in base editing has been done in transformed cells lines, he says. But he wanted to work in cancer cells, in organoids and in vivo. "The organoids are actually the reason we got into base editing to begin with," says Dow, to explore specific mutations in a few key cancer genes.

When working with CRISPR-Cas9 to mutate the $A p c$ gene, where mutations in colon cancer are commonly found, Dow and his team saw "some unexpected things," he says, such as large deletions. Rather than offtarget mutations, he says, these were "errant on-target" mutations. "The large deletions were giving us all sorts of different protein products," he says.

As base editing emerged, the Dow lab tried the early editors but found that they

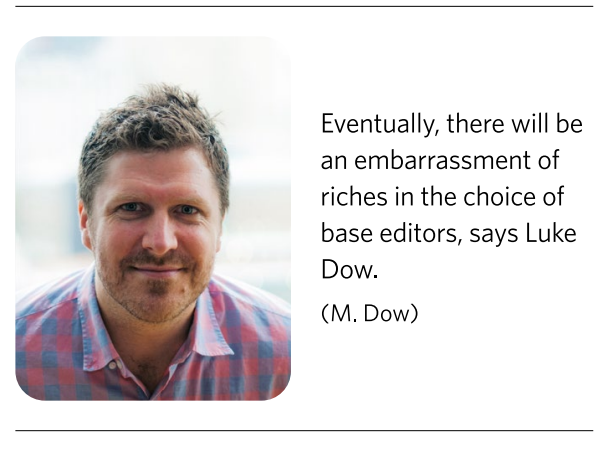

failed in embryonic stem cells and organoids. One issue was that base editing sometimes leads to indels rather than mutations, which can be linked to the different performance of nickases versus Cas9. When using the same gRNA, they experienced a much higher frequency of indels in some cell lines than in others. "Our working hypothesis is that these cells have different DNA damage repair mechanisms," says Dow, which leads to different levels of mutation versus deletion. This effect is reduced with second- and thirdgeneration base editors but is not completely eliminated, he says.

When cloning lentiviral constructs for transfection, they had noticed that Cas9n DNA in the base editor BE3 was not expressed well in mammalian cells. They optimized protein expression, which led to a range of reengineered enzymes that are better at gene targeting. And they enhanced the nuclear targeting to ease delivery of ribonuclear particles. The improvements let them generate the single-nucleotide variants they sought. They have tested their base editors in mouse and human cell lines, as well as intestinal organoids to model colon cancer. They also made in vivo somatic edits to bases in the DNA of liver cells in adult mice.

CTNNB1 mutations play a role in a Wnt pathway involved in liver tumors. The lab edited bases in this gene to drive tumor formation in live mice and model cancer. They activated Wnt and promoted tumor growth, which has not been possible with classic CRISPR-Cas. "It's very hard to engineer these types of situation with Cas9 alone," says Dow. The result would be insertions and deletions instead of activating point mutations.

In this project, Dow and his team used two gRNAs to create an inactivating mutation in $A p c$ and an activating mutation in PI-3 kinase "We use that example just to show that simultaneously you can activate one gene and inactivate another with the same tool, just by changing the guide RNA," says Dow.
Since publication of his paper, labs have reached out to Dow, noting their own struggles. Base-editing efficiency appears to differ between cell types and cell lines, he says, and this variability is linked to how well the enzymes are expressed in a given cell. Now that his lab has built a "micro-infrastructure," they plan to build new base editors or update existing ones as new approaches emerge, says Dow. For example, into their 'backbones' the lab has cloned versions of base editors with engineered deaminases from the Joung lab. Iteration is how he and his team hunt for the base editors they need. Speaking more generally about the base-editing field, he says, "Eventually, we are going to have sort of an embarrassment of riches in choice."

Dow especially looks forward to tunable base editing. "You can turn it on, create the mutation and then turn it off again," he says. During his postdoctoral fellowship, he made inducible and reversible shRNA mice. For base editing, this experience offered a lesson about targeted mutations. Tuned mutations could let labs avoid potential issues associated with continuous expression of APOBEC enzymes, which can damage a cell or provoke an immune reaction. By making a strain that already carries the Cas9, one can perhaps avoid immunogenicity issues, he says.

When CRISPR was first developed, Dow says, it was common to describe it as a precise tool. "But that sort of opened our eyes to the fact that you could now start picking apart the genome with single-base resolution, and we realized CRISPR wasn't so sharp after all," he says. Being able to make targeted point mutations is new. "Five years ago this was total science fiction."

\section{Vivien Marx \\ Technology editor for Nature Methods. \\ e-mail:v.marx@us.nature.com}

Published online: 1 October 2018

https://doi.org/10.1038/s41592-018-0146-4

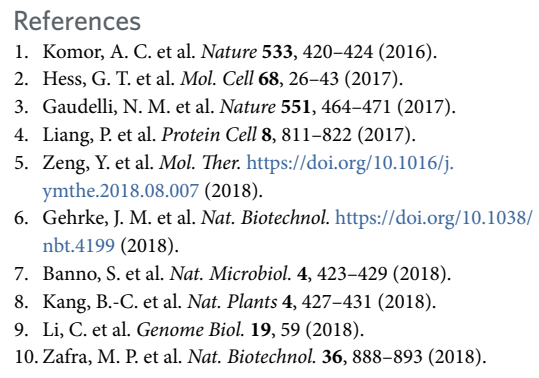

References

1. Komor, A. C. et al. Nature 533, 420-424 (2016).

2. Hess, G. T. et al. Mol. Cell 68, 26-43 (2017).

4. Liang, P. et al. Protein Cell 8, 811-822 (2017).

5. Zeng, Y. et al. Mol. Ther. https://doi.org/10.1016/j. ymthe.2018.08.007 (2018).

8. Kang, B.-C. et al. Nat. Plants 4, 427-431 (2018).

10. Zafra, M. P. et al. Nat. Biotechnol. 36, 888-893 (2018). 